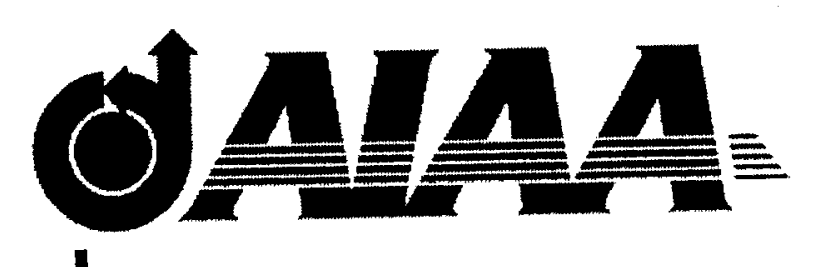

AIAA 2001-1065

\title{
Shape Optimization of Supersonic Turbines Using Response Surface and Neural Network Methods
}

Nilay Papila and Wei Shyy

University of Florida, Gainesville, FL

and

Lisa W. Griffin and Daniel J. Dorney

NASA Marshall Flight Center, Huntsville, AL

\section{9th AIAA Aerospace Sciences Meeting and Exhibit 8-11 January 2001 / Reno, Nevada}


AIAA 2001-1065

\title{
SHAPE OPTIMIZATION OF SUPERSONIC TURBINES USING RESPONSE SURFACE AND NEURAL NETWORK METHODS
}

\author{
Nilay Papila*, Wei Shyy", Lisa Griffin‡, and Daniel J. Dorneył \\ "Department of Aerospace Engineering, Mechanics \& Engineering Science \\ University of Florida, Gainesville, FL \\ ‡NASA Marshall Flight Center, Huntsville, AL
}

\begin{abstract}
Turbine performance directly affects engine specific impulse, thrust-to-weight ratio, and cost in a rocket propulsion system. A global optimization framework combining the radial basis neural network (RBNN) and the polynomial-based response surface method (RSM) is constructed for shape optimization of a supersonic turbine. Based on the optimized preliminary design, shape optimization is performed for the first vane and blade of a 2-stage supersonic turbine, involving $O(10)$ design variables. The design of experiment approach is adopted to reduce the data size needed by the optimization task. It is demonstrated that a major merit of the global optimization approach is that it enables one to adaptively revise the design space to perform multiple optimization cycles. This benefit is realized when an optimal design approaches the boundary of a pre-defined design space. Furthermore, by inspecting the influence of each design variable, one can also gain insight into the existence of multiple design choices and select the optimum design based on other factors such as stress and materials considerations.
\end{abstract}

\section{INTRODUCTION}

Turbine performance directly affects engine specific impulse, thrust-to-weight ratio, and cost in a rocket propulsion system. In the last three decades, supersonic turbines have not been designed for rocket propulsion in the U.S. There are growing interests to reconsider this technology for space transport. Designing a multistage turbine is a labor-intensive task because of the substantial number of variables involved in the problem. Clearly, a formal optimization methodology will be valuable to help meet the design goals by maximizing the performance objective while addressing the structures and materials considerations. A number of papers using approaches such as sensitivity evaluations (Refs. [1]- [5]), genetic algorithms (Refs. [6]- [8]), or response surface methods (Refs. [9]-[12]) have been published in this area. In this work, a global optimization methodology, based on the radial-basis neural networks (RBNN) and the polynomialbased response surface method (RSM) and under development by the authors (Refs. [13]- [14]), is employed to facilitate design optimization for supersonic turbines intended for the reusable launch vehicle (RLV) applications.

We select to use the global optimization technique because it has several advantages when compared to local methods [15]: (1) they do not require calculation of the local sensitivity of each design variable, (2) they can utilize the information collected from various sources and by different tools, (3) they offer multicriterion optimization, (4) they can handle the existence of multiple design points and trade-offs, (5) they easily perform tasks in parallel, and (6) they can often effectively filter the noise intrinsic to numerical and experimental data. Among alternative global approximation techniques, the RSM has gained the most attention due to its flexibility in handling different types of information [16]. The RSM expresses the objective and constraint by simple functions, often polynomials, which are fitted to the selected points. One can use RBNN-based and polynomial-based RSM techniques to model the relationship between design variables and objective/constraint functions of the overall approach. In the present work, the RBNN is employed to supply additional data to help construct improved polynomial representation of the response surface. In other words, RBNN feeds data into polynomials before the optimization task is conducted. Such a practice has been shown to be beneficial (Refs. [12]- [14]), and can help assess the adequacy of the response surface model when there is insufficient amount of information available.

With the aid of the global optimization technique, both preliminary and detailed shape designs are considered. The main purpose for preliminary optimization is to determine the optimum configuration in terms of the number of stages, sizing, RPM and compatibility between operating variables [17]. Based on this exercise, shape optimization is conducted for each vane and blade. The preliminary design relies heavily on simplified one-dimensional and semi-empirical formulas, whereas the shape optimization depends largely on the 
Navier-Stokes computational fluid dynamics (CFD) simulations.

In any optimization process, and especially with global optimization techniques, the amount and the distribution of data required is a critical issue. To help address this question, we have investigated the issues related to the design of experiments (DOE). Typically, the face centered composite design (FCCD)[18] is employed. This approach can be quite costly as the number of design variables increases. Additional criteria can be established to help improve the efficiency and effectiveness of the construction of the response surface by employing the concept of orthogonal arrays (OA)[19] and D-optimality [20]. These methods will be discussed in the following sections.

\section{APPROACH}

As shown in Figure 1, the entire optimization process can be divided into three parts: (1) data generation, (2) polynomial or NN generation phase for establishing an approximation, and (3) optimizer phase. In the first phase, the representation of design space is decided. In the second phase, polynomials or NN (or combined) models are generated with the available training data set. Finally, in the third phase the optimizer uses the polynomial or NN approximations during the search for the optimum until the final converged solution is obtained.

The optimization technique follows our previous works for optimizing fluid machinery, such as diffuser, injector, and airfoil, as presented in Refs. [13]-[15], [17], and [21]-[23]. The neural network technique and the polynomial RSM are integrated to offer enhanced optimization capabilities by Shyy et al. [14]. Optimization of a supersonic turbine for preliminary design, using the polynomial RSM, is presented by Papila et al [17]. Papila et al. [21] investigated the effect of data size and relative merits between polynomial and neural networks-based RSM in handling varying data characteristics. Issues related to numerical noises and the interaction between CFD models and RSM are addressed by Madsen et al. [22]. In Tucker et al.[23], a first effort is made to apply RSM for injector optimization. Refs. [13]\&[14] offers a comprehensive update of the concepts and applications of the global optimization method, including the above mentioned examples.

\subsection{Overview of the Response Surface Literature}

Polynomial-based response surface techniques (parametric regression), when compared to neural network (NN)-based RSM (non-parametric regression), has an advantage in the ease of computing since finding the polynomial coefficients is a linear regression process while finding the weights associated with the neurons of a $\mathrm{NN}$ is, in general, a non-linear regression process. The linearity decreases the computational cost and provides an advantage when the simulations have substantial amount of numerical noise. However, it is possible to design a linear NN model. The RBNN is such an example. In terms of the ability of filtering noise from experimental data, polynomial-based RSM has certain advantages over NN's. However, if the number of neurons used to design the $\mathrm{NN}$ is not the same as the data, then, by definition, filtering is also performed by the NN. When it comes to handling complex functions, neural networks are more flexible to fit complex functions. This advantage is particularly noticeable if the level of numerical noise is low.

A growing number of papers have been published to combine $\mathrm{NN}$ and polynomial-based RSM approximations, see e.g., Refs. [11]-[14] and [24]-[25]. For example, the work done by Rai and Madavan [11]\& [24]-[25], Madavan et al. [12], and Shyy et al. [14] suggest that NN can be effectively used to supplement the existing training data to help generating a more accurate polynomial. RBNN may lack satisfactory filtering properties in some cases as stated by Papila et al. [21] and Vaidyanathan et al. [28]. However, once trained, RBNN can generate additional design data easily to feed the polynomial-based RSM. This approach is also employed in this work.

\subsection{Radial Basis Neural Networks (RBNN)}

Neural networks are massively parallel computational systems comprised of simple nonlinear processing elements with adjustable interconnections [29]. Neural networks simulate human functions such as learning from experience, generalizing from previous to new data, and abstracting essential characteristics from inputs containing irrelevant data [30]. The predictive ability of the network is stored in the inter-unit connection strengths called weights obtained by a process of adaptation to, or learning from, a set of training patterns. Training of a network requires repeated cycling through the data and continues until the error target is met or until the maximum number of neurons is reached.

This article focuses on RBNN, which is a multilayer network with hidden layers of radial-basis function and a linear output layer. Radial-basis functions (RBF) are activation functions of which the response decreases or increases monotonically with distance between the input and the RBF's center. The distance between two points is determined by the difference of their coordinates and by a set of parameters [31].

The main advantages of RBNN approach is the ability of reducing the computational cost due to the linear nature of RBNN [32]. RBNN is a linear model since the basis functions, $h$ in Eqn. 1, and any parameters, which they might contain, are fixed through the training process. 


$$
f(x)=\sum_{j=1}^{m} w_{j} h_{j}(x)
$$

where $w$ is the coefficients of the linear combinations or weights and $h$ is the basis functions.

In non-parametric regression or supervised learning with linear models, least squares principle that minimizes the sum-squared errors can be applied for training and then $m$ unknown weights can be solved based on the mean square error of the training set. However, the criterion of the mean square error of the training set is unlikely to achieve reasonable results for predicting the unknown input. For this purpose so called test data should be used when selecting the model. This is the basic form of cross-validation [31]\& [32]. In this study, the RBNN model is designed by using the functions developed by Orr [31]-[33] for Matlab [34]. Among several choices given by Orr [31], the RBNN method based on ridge regression [32] is used since it can optimize the RBF widths to improve the data fitting capability. In order to choose between competing models, the maximum marginal likelihood method [33] is used as a model selection criteria.

\subsection{Polynomial-Based Response Surface Techniques}

The polynomial-based RSM models the system with assumed order and unknown coefficients. The solution for the set of coefficients that best fits the training data is a linear least square problem [18], and so it is computationally straightforward. The linearity also allows us to use statistical techniques such as DOE to find efficient training sets. Statistical techniques are also available for identifying polynomial coefficients that are not well characterized by the data. These coefficients are discarded in order to improve the predictive capability of the polynomial. Finally, these statistical tools allow us to estimate the error of the polynomial-based model at points not used for training, that is, its prediction error.

In this study, the response surfaces are generated by standard least-squares regression using JMP [35], a statistical analysis software having a variety of statistical analyses functions in an interactive manner. The global fit and prediction accuracies of the response surfaces are assessed through statistical measures such as the t-statistic and the root mean square error ( $r m s$-error) variation.

\subsection{Design of Experiment}

In order to minimize the effect of the noise on the fitted polynomial and neural network, and to improve the representation of the design space, the design of experiments (DOE) procedure can be used to select the data to be used in construction of the response surfaces or training of the RBNN. There are a number of different designs of experiment techniques in the literature, as reviewed in Ref. [36]. Issues related to our approach are reviewed in Ref. [17], and will not be repeated here. In this study, face centered composite design (FCCD), orthogonal arrays (OA), and D-optimal design methods are used to select the effective design space. The FCCD is widely used for fitting second-order response surfaces [18]. It selects design points at the corners of the design space, center of the faces and the center of the design space. Therefore, FCCD yields $\left(2^{N}+2 N+1\right)$ points, where $N$ is the number of design variables. On the other hand, an orthogonal array is a fractional factorial matrix that assures a balanced comparison of levels of any factor or interaction of factors [19]. Because the points are not necessarily at vertices, orthogonal array tools can be more robust than the facecentered cubic design. Based on the design of experiments theory, OA can significantly reduces the number of experimental configurations [21]. Finally, the D-Optimal design minimizes the generalized variance of the estimates that is equivalent to maximizing the determinant of the moment matrix [18]. The D-Optimal Design approach makes use of the knowledge of the properties of polynomial model in selecting the design points. This criterion tends to emphasize the parameters with the highest sensitivity [36].

\subsection{Optimization Procedure}

In the optimization process, the set of design variables is selected to form a design space. The present optimization problems are constrained, which can be formulated as $\min \{f(x)\}$ subject to $l b \leq \mathrm{x} \leq u b$, where $l b$ is the lower boundary vector and $u b$ is the upper boundary vector of the design variables vector $x$. Since the goal is to maximize objective function $f(x)$ can be written as $-g(x)$, where $g(x)$ is the objective function. Additional linear or nonlinear constraints can be incorporated.

The main focus in the present work is the interplay between the number of design variables and the predictive capability and input requirement of the RSM. Microsoft Excel Solver [37] is used as an optimization toolbox together with the polynomial response surface technique throughout the work. Solver applies generalized reduced gradient method [38] to find the maximum or minimum of a function with given constraints.

\section{RESULTS AND DISCUSSION}

\subsection{Preliminary Design}

For preliminary design stage, we have considered single-, 2 - and 3-stage turbines with the number of design variables increasing from 6 to 11 then to 15 , in accordance with the number of stages. The preliminary design parameters for single-, 2- and 3- stage turbines are shown in Table 1.

The preliminary design data is obtained by using a computer code: meanline [39]. The meanline code 
performs one-dimensional (1D) analyses that employ loss correlations gleaned from experimental databases. It predicts performance, calculates gas conditions and velocity triangles, generates a flowpath elevation, and estimates the turbopump weight. It also provides an initial spanwise distribution of row exit angle based on the assumption of constant axial velocity and conservation of radial momentum.

The optimum preliminary design is obtained by using polynomial-based RSM coupled with a meanline analysis. For this design stage, the purpose is to maximize overall T-S Efficiency, $\eta$, and minimize overall weight, $W$, simultaneously for each number of stages case, 1, 2 or 3. A function to correlate turbine efficiency and turbopump weight to payload capacity is derived from system models.

As discussed in Ref [17], in order to ascertain required predictive capability of the RSM, a two-level domain refinement strategy has been adopted. The accuracy of the predicted optimal design points based on this approach is improved. The results of payload increment based optimization are illustrated in Figure 2 and Table 2 for 1, 2, and 3-stage designs. From this figure, it can be observed that, the optimum $\Delta$ pay increase from single-stage to 2-stage, whereas it decreases from 2-stage to 3-stage. According to the formula for $\Delta$ pay, the improvement in efficiency can not compensate the penalty from higher weight as the number of the stage increases. The 2 -stage turbine gives the best $\Delta$ pay improvement, indicating that it is the optimum configuration (Table 3 ).

\subsection{Detailed Shape Design}

In detailed design, the shapes of the vanes and blades are generated. To generate optimum detailed designs, CFD analysis, RBNN and polynomial-based RSM are used. Ideally, the detailed optimization would be conducted in one step all row optimized simultaneously. However, the sheer number design variables made this approach impractical. Therefore, first the mean airfoil contours of each component are optimized. Airfoil contours are generated using a geometry generator developed for this task that could read a matrix design variables, generate and plot the airfoil, and write a preliminary input file for the CFD grid generator [39]. Because a large amount of loss in a supersonic turbine can be attributed to interactions between the first two rows, unsteady CFD calculations are performed for the stage, running parametrics on the vane first with the baseline blade, and then performing the blade parametrics with an optimized vane. For detailed design, CFD calculations are performed using WILDCAT, a parallelized, unsteady, quasi threedimensional (3D) Navier-Stokes code that utilizes moving grids to simulate the rotor motion, is used (Refs. [6] \& [39]-[40]). This paper presents the progress made to date for the first vane and blade design optimization.

\section{(a) The $1^{\text {st }}$ Vane}

There are 7 design parameters for detailed design of the first vane (Table 4). These are leading edge (LE) pressure side height/axial chord, $H / L$, uncovered turning, Unc.Turn, and Bezier curve control handles, $L I, L 3 F$, $L 3 R, L A$, and $L 5$ shown in Figure 3 . For this case, FCCD yields 143 possible design points. After the addition of 6 more levels as shown Figure 4, 437 design points are obtained. Using D-optimality 203 design points are selected to generate CFD solutions.

The objective for detailed design optimization is the stage total-to-total efficiency, $\eta_{T-T}$. The minimum thickness that should be greater or equal to 0.055 is the only design constraint of the first vane optimization.

A three-cycle optimization strategy has been adopted for the first vane. The statistical summary of the response surface models resulting from the three cycles are shown in Table 5. In Cycle 1, we have employed response techniques using reduced cubic polynomial model to identify the optimal design points. The design variables corresponding to the optimized vane shape are shown in Figure 5. It is observed that the optimum design approaches the boundary of the design space. Naturally, it seems fruitful to consider expanding the design space to further improve the design by generating additional information in an extended region. Accordingly, in Cycle 2 , the design space is modified by extending the ranges of two design parameters, namely, L4 and L5. As shown in Table 6, 16 new data are added to the database. The reduced cubic polynomial response surface is again constructed based on the extended design space.

In Cycle 3, a technique based on the $N N$ Enhanced RSM is developed. The procedures can be summarized as follows:

- RBNN is constructed for the subset of 177-point from the 203-data from Cycle 1 and is tested by using the rest of 26-data.

- The trained RBNN is used to predict 87 additional data $(50-\mathrm{OA}$ data +37 previously excluded FCCD data). These data are added to the existing data set of 219-point used in Cycle 2.

- In Cycle 3, with the enhanced data, the reduced cubic polynomial response surface is constructed and tested by using the same 26-data.

- The optimum design is determined for the enhanced design space in Cycle 3.

The results of the optimal designs from all cycles and the CFD results for Cycle $2 \& 3$ are summarized in Table 7 . It is noted that the second and third optimization cycles lead to a converged optimal design. Based on the diagnosis (e.g., contour plots), it appears that the response surface is quite flat with respect to certain design variables (e.g., L4 and L5), in regions close to the optimal 
designs. Consequently, small deviations in other variables can cause noticeable shifts. However, there are multiple design points, including the best data point from the CFD runs, which offer essentially the same performance. This outcome means that other considerations such as stress distribution and manufacturing difficulties can be usefully incorporated into the final selection stage.

Figure 5 shows the comparison of the optimal design variables (normalized in to $(-1,+1)$ range) for reduced cubic models for all design cycles. Figure 6 shows that the designs converge to the same configuration between Cycles 2 and 3 . On the other hand, as shown in Figure 7, there are multiple design points which exhibit essentially the same performance (within the modeling uncertainty), albeit with much different geometries. To help better understand these observations, we assess the sensitivity of the two geometric variables (L4 and L5) in Figure 8, which shows that the performance of the vane is very insensitive to L5. The two shapes shown in Figure 7 are mainly caused by L5, which explains why two very different vane shapes give essentially the same performance. From the structural point of view, the value of L5 to produce thicker profile is preferable. This example demonstrates that the global optimization technique enables one to compare designs based on insight into the entire design space, which allows a suitable selection to be made with additional factors considered. Figure 9 shows the Mach contours of the two vanes shown in Figure 7.

\section{(b) The $\mathbf{1}^{\text {st }}$ Blade}

There are 11 design parameters for detailed design of the first blade (Table 8). These are LE pressure side height/axial chord $(H / L)$, Leading edge metal angle (BetaI), uncovered turning (Unc.Turn), and 6 different Bezier curve control handles $(L 1, L 2 F, L 3 R$, $L A, L 5$, and $L 7$, see Fig. 3), channel convergence ratio (ChanCR), and the circle factor (circfac). Again, the stage total-to-total efficiency $\eta_{T-T}$, is selected as the objective function to be maximized for the blade design. For the first blade, 3 different constraints need be satisfied: (1) minimum thickness that should be greater or equal to trailing edge diameter, (2) $L I+L 2 F+\left(1.9{ }^{*}\right.$ circfac $)$ should be smaller than 2.7 , and (3) the suction side radius of curvature must be greater than 5 times of the trailing edge radius.

For this case, a 5-level OA-based procedure has been adopted to select 250-data for the CFD code to generate solutions. A software tool developed by Owen [19] is used for constructing OA designs. Since these points are located at either the edge or the center of the design space, additional 23 FCDD data at the center of the faces are added. Finally, 38-data along the main diagonal of the design space is generated. In short, a total of 311 possible design points are selected to generate the CFD solutions. However, only 139 out of the 250 OAselected data, 21 out of the 23 FCCD-selected data, and 17 out of the 38 diagonal-based data are obtained from the CFD tool. Therefore, only a total of 177 possible design points, out of the 311 cases, have been collected. The cases are not completed because the airfoil shapes are unrealistic, which cause either excessively high losses, or prevent the grid generation process from running appropriately. Figure 10 illustrates the distribution of both types of cases. As one can see from the figure, the difficulty is along the diagonal ends as well as on the edges of the design space. In Figure 11, a sample blade shape from the cases for which the CFD code does not converge is illustrated. With only 177-data generated for 11 design variables, RBNN is used to produce additional information, as described below.

- RBNN is trained based on the 160 data points from the above-mentioned $177 \mathrm{CFD}$-generated data, with the remaining 17 points reserved to tune the configuration of the neural networks.

- The trained RBNN is used to generate additional 2204 possible design points. 2070 of them are selected using FCCD data modified as shown in Figure 12 and the rest 134 of them are the cases for which CFD code does not converge.

- Full quadratic and reduced cubic response surface models are constructed for the first blade. A total of 38data are used to test both models; of which, 17 are from the CFD runs (originally selected as the test data), and 21 are from the $\mathrm{NN}$-enhanced data. The statistical summary for both models, with and without NN-enhanced data, is shown in Table 9.

- In the course of performing the optimization task, it is realized that out of the 11 design variables, 5 of them, responsible for the shape in the rear portion of the upper blade surface, can be optimized first, because various plausible shapes converge to the similar values of them. Accordingly, one can reduce the design space from 11 to 6 variables. The revised design space is summarized in Table 10, which shows that the 5 fixed design variables are of single values, while the other 6 variables have different ranges. Two designs are presented, as shown in Table 11 and Figure 13. With 6 design variables fixed, the two blade shapes yield very comparable performance. Their shapes in the upper rear portion are, as expected, virtually the same. On the other hand, their front portions are noticeably different, with Blade-1 exhibiting more variations than Blade-2. Again, with the insight offered by the global model, we have an opportunity to evaluate not only the sensitivity of each design variable, but also additional trade-offs between comparable designs. 


\section{CONCLUDING REMARKS}

A methodology to improve the supersonic turbine performance has been developed for preliminary and detailed design. A global optimization framework combining the RBNN and the polynomialbased RSM is constructed. Based on the optimized preliminary design, shape optimization is performed for the first vane and blade of an optimized 2-stage supersonic turbine. The results obtained can be summarized as follows:

(1) The design of experiment approach is critical in reducing the data size needed by the optimization task.

(2) A major merit of the global optimization approach is that it enables one to adaptively revise the design space to perform multiple optimization cycles.

(i) As evidenced by the three optimizationcycles conducted for the first vane, capabilities to adaptively refine the optimization scope and procedure can be critical when an optimal design approaches the boundary of a pre-defined design space.

(ii) For the first blade, the optimization process indicates that 5 out of the 11 design variables can be chosen first, leaving the design space to reduce to have 6 variables. Based on the reduced set of design variables, the optimization task can be handled more effectively.

(3) By inspecting the influence of each design variable, one can also gain insight into the existence of multiple design choices and select the optimum design based on other factors such as stress and materials considerations.

(4) The NN-enhanced RSM helps to improve the accuracy of the response surface, and allows the optimization task to be conducted with smaller number of CFD runs.

\section{ACKNOWLEDGEMENTS}

This work has been supported by NASA Marshall Space Flight Center. We appreciate the useful discussions with Frank Huber (Riverbend Design Services) and Ken Tran (Boeing-Rocketdyne).

\section{REFERENCES}

[1] Lee, S.-Y. and Kim, K.-Y., "Design Optimization of Axial Flow Compressor Blades with Three- Dimensional Navier-Stokes Solver," Proceedings of ASME Turbo Expo 2000, Germany, May 8-11, 2000.

[2] Baysal, O., and Ghayour, K., "Continuous Adjoint Sensitivities for General Cost Functionals on Unstructured Meshes in Aerodynamic Shape Optimization, " Proceedings of 7 th AIAA/USAF/NASA/ISSMO Symposium on
Multidisciplinary Analysis and Optimization, St. Louis, MO, AIAA 98-4904 CP September 2-4, 1998.

[3] Sørensen, D. N., Thompson, M.C., and Sørensen, J. N., "Toward Improved Rotor-Only Axial Fans-Part II: Design Optimization for Maximum Efficiency," Journal of Fluids Engineering, Vol. 122, pp.324-329, 2000.

[4] Janus, J. M. and Newman III, J. C., " Aerodynamic and Thermal Design Optimization for Turbine Airfoils," 38th AIAA Aerospace Sciences Meeting and Exhibit, Reno, Nevada, AIAA 2000-0840, 2000.

[5] Yiu, K. F. C. and Zangeneh, M., "A $3 D$ Automatic Optimization Method for Turbomachinery Blade Design," to be published in AIAA Journal of Propulsion and Power, 1999.

[6] Snodgrass, R., Dorney, D. J., Sandgren, E., and Merz, L. F., "Multi-objective Shape Optimal Design of a Supersonic Turbine," 8th AIAA/USAF/NASA/ISSMO Symposium on Multidisciplinary Analysis and Optimization Conference, Long Beach, CA, AIAA 20004860, 2000.

[7] Wang, S., Fan, H., and Xi, G., "Performance Comparison Study of an Improved Genetic Algorithm Applied to an Aerodynamic Design," The Second International Symposium On Fluid Machinery And Fluid Engineering, October, Beijing, China, 2000, pp.88-95.

[8] Pierret, S., " Three- Dimensional Blade Design by means of an Artificial Neural Network and Navier-Stokes Solver," Turbomachinery Blade Design Systems Lecture Series 1999-02, 1999.

[9] Huang, S. Y., Miller, L. S., and Steck J. E., "An Exploratory Application of Neural Networks to Airfoil Design," 32nd AIAA Aerospace Sciences Meeting and Exhibit, Reno, Nevada, AIAA 94-0501, 1994.

[10] Burman, J., Gebart B., and Martensson, H., "Development of a Blade Geometry Definition with Implicit Design Variables," 38th AIAA Aerospace Sciences Meeting and Exhibit, Reno, Nevada, AIAA 2000-0671, 2000.

[11] Rai, M.M. and Madavan, N.K, "Improving the Unsteady Aerodynamic Performance of Transonic Turbines Using Neural Networks," 38th AIAA Aerospace Sciences Meeting and Exhibit, Reno, Nevada, AIAA 2000-0169, 2000.

[12] Madavan, N.K, Rai, M.M. and Huber, F. W., "Neural Net-Based Redesign of Transonic Turbines for Improved Unsteady Aerodynamic Performance," AIAA/SAE/ASME/ASEE 35th Joint Propulsion Conference, AIAA 99-2522, June 20-24., 1999.

[13] Shyy, W., Papila, N., Vaidyanathan, R., and Tucker, P. K., "Global Design Optimization for Aerodynamics and Rocket Propulsion Components," to be published in Journal of Progress in Aerospace Sciences, 2000. 
[14] Shyy, W., Tucker, P.K. and Vaidyanathan, R., "Response Surface and Neural Network Techniques for Rocket Engine Injector Optimization," AIAA/SAE/ASME/ASEE 35th Joint Propulsion Conference, AIAA 99-2455, June 20-24, 1999.

[15] Shyy, W., Papila, N., Tucker, P. K., Vaidyanathan, R., and Griffin L., "Global Design Optimization for Fluid Machinery Applications," The Second International Symposium On Fluid Machinery And Fluid Engineering, October, Beijing, China, 2000, pp.1-10.

[16] Sobieszczanski-Sobieski, J. and Haftka, R.T., "Multidisciplinary aerospace design optimization: survey of recent developments," Structural Optimization, Vol. 14, pp.1-23, 1997.

[17] Papila, N., Shyy, W., Griffin, L., Huber,F., and Tran, K., "Preliminary Design Optimization For A Supersonic Turbine For Rocket Propulsion," 36th AIAA/ASME/SAE/ASEE Joint Propulsion Conference and Exhibit, Huntsville, AL, AIAA 2000-3242, 2000.

[18] Myers, R. H., and Montgomery, D. C., Response Surface Methodology - Process and Product Optimization Using Designed Experiments, New York: John Wiley \& Sons, Inc., 1995.

[19] Owen, A., "Orthogonal Arrays for: Computer Experiments, Integration and Visualization," Statistica Sinica, Vol. 2, No.2, pp. 439-452, 1992.

[20] Unal, R., Lepsch, R. A., and McMillin, M. L., "Response Surface Model Building and Multidisciplinary Optimization using D-Optimal Designs," $7^{\text {th }}$ AIAA/USAF/NASA/ISSMO Symposium on Multidisciplinary Analysis and Optimization, AIAA 984759, 1998.

[21] Papila, N., Shyy, W., Fitz-Coy, N. and Haftka, R.T., "Assessment of Neural Net and Polynomial-Based Techniques for Aerodynamic Applications, " 17th Applied Aerodynamics Conference, Norfolk, VA, AIAA 99-3167, 1999.

[22] Madsen, J.I., Shyy, W. and Haftka, R.T, "Response Surface Techniques for Diffuser Shape Optimization," AIAA Journal, Vol. 38, pp. 1512-1518, 2000.

[23] Tucker, P.K., Shyy, W. and Sloan, J.G., "An Integrated Design/Optimization Methodology for Rocket Engine Injectors," AIAA/SAE/ASME/ASEE 34th Joint Propulsion Conference, AIAA-98-3513, 1998.

[24] Rai, M.M. and Madavan, N.K, "Application of Artificial Neural Networks to the Design of Turbomachinery Airfoils," 36th AIAA Aerospace Sciences Meeting and Exhibit, Reno, Nevada, AIAA 2000-0169, 1998.

[25] Rai, M.M. and Madavan, N.K, "Aerodynamic Design Using Neural Networks," 7th AIAA/USAF/ NASA/ISSMO Symposium on
Multidisciplinary Analysis and Optimization, St. Louis, AIAA 98-4928, 1998.

[26] Carpenter, W.C. and Barthelemy, J.-F.M, "A comparison of Polynomial Approximations and Artificial Neural Nets as Response Surface," Structural Optimization, Vol. 5, pp.166-174, 1993.

[27] Nikolaidis, E., Long, L., and Ling, Q., "Neural Networks and Response Surface Polynomials for Design of Vehicle Joints," 39th AIAA/ASME/ ASCE/AHS/ASC Structures, Structural Dynamics, and Materials Conference, Long Beach, California, AIAA-98$1777,1998$.

[28] Vaidyanathan, R., Papila, N., Shyy, W., Tucker, P.K., Griffin, L. W., Fitz-Coy, N. and Haftka, R.T, "Neural Network-Based and Response SurfaceBased Optimization Strategies for Rocket Engine Component Design," 8th AIAA/USAF/NASA/ISSMO Symposium on Multidisciplinary Analysis and Optimization, Long Beach, CA, 2000.

[29] Kosko, B, Neural Networks and Fuzzy Systems: A Dynamical Systems Approach to Machine Intelligence, Prentice Hall, 1992.

[30] Greenman, R. M., "2-D High-Lift Aerodynamic Optimization Using Neural Networks," NASA TM-1998-112233, June 1998, pp. 146, 1998.

[31] Orr, M. J. L., "Matlab Functions for Radial Basis Function Networks," Institute for Adaptive and Neural Computation Division for Informatics, Edinburgh University, EH 9LW, Scotland, UK, 1999 (http://www.anc.ed.ac.uk/ mjo/rbf.html).

[32] Orr, M. J. L., "Introduction to Radial Basis Function Networks," Centre for Cognitive Science, Edinburgh University, EH 9LW, Scothland, UK, 1996 (http://www.anc.ed.ac.uk/ mjo/rbf.html).

[33] Orr, M. J. L., "Recent Advances in Radial Basis Function Networks," Technical Report, Institute for Adaptive and Neural Computation Division for Informatics, Edinburgh University, EH 9LW, Scotland, UK, 1999 (http://www.anc.ed.ac.uk/ mjo/rbf.html).

[34] Matlab: The language of technical Computing, Version 5.3.0.10183 (R110, The Math Works Inc (1984-1999).

[35] JMP version 3, Statistics And Graphics Guide, SAS Institute Inc., 1998.

[36] Haftka, R.T., Scott, E.P., and Cruz, J.R., "Optimization and Experiments: A Survey," Applied Mechanics Reviews, 51(7), pp. 435-448, 1998.

[37] Microsoft Excel Solver 97, Microsoft Corporation (1985-1996).

[38] Ladson, L.S., Waren, A. D., Jain, A., and Ratner, M., "Design and Testing of a Generalized Reduced Gradient Code for Non-linear Programming," ACM Transactions on Mathematical Software, Vol. 4, No. 1, pp.51-56, 1978.

[39] Griffin, L. W. and Dorney, D. J., "RLV Turbine Performance Optimization," 12 th Annual 
Propulsion Engineering Research Center (PERC) Symposium, Cleveland, OH, 2000.

[40] Griffin, L. W. and Dorney, D. J., "Simulations of the Unsteady Flow Through the Fastrac Supersonic Turbine," ASME Journal of Turbomachinery, Vol.122, No.2, pp.225-233, 2000.

Table 1. Preliminary Design Variables (All geometrical variables are normalized by baseline values) (a) Single-stage turbine

\begin{tabular}{|l|c|c|}
\hline Design Variable & Lower Limit & Upper Limit \\
\hline Mean diameter & 0.5 & 1.5 \\
\hline$R P M$ & 0.7 & 1.3 \\
\hline Blade annulus area & 0.7 & 1.3 \\
\hline Vane axial chord & 0.4 & 1.7 \\
\hline Blade axial chord & 0.3 & 1.1 \\
\hline Stage reaction. & 0.0 & 0.5 \\
\hline
\end{tabular}

(b) Additional variables for 2-stage turbine

\begin{tabular}{|l|c|c|}
\hline Design Variable & Lower Limit & Lower Limit \\
\hline 1 st blade height & 0.9 & 1.5 \\
\hline $2^{\text {nd }}$ vane axial chord & 0.3 & 1.8 \\
\hline $2^{\text {nd }}$ blade axial chord & 0.3 & 1.1 \\
\hline $2^{\text {nd }}$ stage reaction & 0.0 & 0.5 \\
\hline $1^{\text {st }}$ stage work fraction & 0.5 & 0.85 \\
\hline
\end{tabular}

(c) Additional variables for 3-stage case

\begin{tabular}{|l|c|c|}
\hline Design Variable & Lower Limit & Lower Limit \\
\hline $3^{\text {rd }}$ vane axial chord & 0.2 & 1.4 \\
\hline $3^{\text {rd }}$ blade axial chord & 0.2 & 1.1 \\
\hline $3^{\text {rd }}$ stage reaction & 0.0 & 0.5 \\
\hline $1^{\text {st }}$ stage work fraction & 0.4 & 0.8 \\
\hline $2^{\text {nd }}$ stage work fraction & 0.1 & 0.3 \\
\hline
\end{tabular}

Table 2. Optimization summary for 1,2 and 3-stage turbine with response surface in refined design space (All output parameters are normalized by the baseline values)

\begin{tabular}{|l|c|c|c|c|}
\cline { 3 - 5 } \multicolumn{1}{c|}{} & $\eta_{\text {opt }}$ & $\mathrm{W}_{\text {opt }}$ & $\Delta$ pay $_{\text {opt }}$ \\
\hline$\Delta$ pay & 1-stage & 0.77 & 0.73 & -0.21 \\
\hline & 2-stage & 1.13 & 1.04 & 0.15 \\
\hline & 3-stage & 1.20 & 1.54 & 0.11 \\
\hline
\end{tabular}

Table 3. Optimum 2-stage Turbine Parameters (All geometrical variables are normalized by baseline values)

\begin{tabular}{|l|c|}
\hline Design Variable & Optimum \\
\hline Mean diameter & 1.1 \\
\hline$R P M$ & 1.0 \\
\hline Blade annulus area & 1.1 \\
\hline 1st blade height & 1.5 \\
\hline $1^{\text {st }}$ Vane axial chord & 1.4 \\
\hline $1^{\text {st }}$ Blade axial chord & 0.7 \\
\hline $2^{\text {nd }}$ vane axial chord & 0.8 \\
\hline $2^{\text {nd }}$ blade axial chord & 0.6 \\
\hline $1^{\text {st }}$ stage reaction & 0.1 \\
\hline $2^{\text {nd }}$ stage reaction & 0.5 \\
\hline $2^{\text {nd }}$ stage work fraction & 0.9 \\
\hline
\end{tabular}


Table 4. Detailed Design Variables for the first vane (All variables are normalized by baseline values)

\begin{tabular}{|l|c|c|}
\hline Design Variable & Lower Limit & Upper Limit \\
\hline H/L & 0.79 & 1.19 \\
\hline Uncovered Turning & -1.20 & -0.20 \\
\hline L1 & 0.36 & 2.00 \\
\hline L3F & 0.44 & 1.76 \\
\hline L3R & 0.25 & 2.00 \\
\hline L4 & 0.05 & 1.25 \\
\hline L5 & 0.13 & 2.00 \\
\hline
\end{tabular}

Table 5. Quality the Polynomial Response Surface Models for the Original (Cycle 1), Extended (Cycle 2) and NNEnhanced (Cycle 3) Design Spaces for the first vane (The mean of response is normalized by the baseline $\eta_{T-T}$ )

\begin{tabular}{|c|c|c|c|c|}
\hline Summary of Fit & RSquare & $\begin{array}{c}\text { RSquare } \\
\text { Adj }\end{array}$ & $\begin{array}{c}\text { Root Mean } \\
\text { Square Error }\end{array}$ & $\begin{array}{c}\text { Observations (or } \\
\text { Sum Wgts) }\end{array}$ \\
\hline $\begin{array}{c}\text { Reduced Cubic Model } \\
\text { (203-data) (Cycle 1) }\end{array}$ & 0.8 & 0.8 & $1.1 \%$ & 203 \\
\hline $\begin{array}{c}\text { Reduced Cubic Model } \\
\text { (219-data) (Cycle 2) }\end{array}$ & 0.8 & 0.8 & $1.1 \%$ & 219 \\
\hline $\begin{array}{c}\text { Reduced Cubic Model } \\
\text { (306-data) (Cycle 3) }\end{array}$ & 0.7 & 0.7 & $1.3 \%$ & 306 \\
\hline
\end{tabular}

Table 6. Comparison of the Original (Cycle 1), Extended (Cycle 2) and NN-Enhanced (Cycle 3) Design Spaces for the first vane

\begin{tabular}{|l|c|c|c|c|c|c|}
\cline { 2 - 7 } \multicolumn{1}{c|}{} & \multicolumn{2}{c|}{ Cycle 1 } & \multicolumn{2}{c|}{ Cycle 2 } & \multicolumn{2}{c|}{ Cycle 3 } \\
\hline Design Variable & Lower Limit & Upper Limit & Lower Limit & Upper Limit & Lower Limit & Upper Limit \\
\hline H/L & 0.79 & 1.19 & 0.79 & 1.19 & 0.79 & 1.19 \\
\hline Uncovered Turning & -1.20 & -0.20 & -1.20 & -0.20 & -1.20 & -0.20 \\
\hline L1 & 0.36 & 2.00 & 0.36 & 2.00 & 0.36 & 2.00 \\
\hline L3F & 0.44 & 1.76 & 0.44 & 1.76 & 0.44 & 1.76 \\
\hline L3R & 0.25 & 2.00 & 0.25 & 2.00 & 0.25 & 2.00 \\
\hline L4 & 0.05 & 1.25 & $\mathbf{0 . 0 1}$ & $\mathbf{1 . 3 5}$ & $\mathbf{0 . 0 1}$ & $\mathbf{1 . 3 5}$ \\
\hline L5 & 0.13 & 2.00 & $\mathbf{0 . 0 1}$ & $\mathbf{2 . 5 0}$ & $\mathbf{0 . 0 1}$ & $\mathbf{2 . 5 0}$ \\
\hline
\end{tabular}

Table 7. Optimum Designs for the Original (Cycle 1), Extended (Cycle 2) and NN-Enhanced (Cycle 3) Design Spaces for the first vane (All design variables and $\eta_{\mathrm{T}-\mathrm{r}}$ are normalized by the baseline values)

\begin{tabular}{|l|c|c|c|c|c|c|c|c|c|c|}
\cline { 3 - 10 } \multicolumn{2}{c|}{} & $\mathrm{H} / \mathrm{L}$ & Unc. Turning & L1 & L3F & L3R & L4 & L5 & $\eta_{\text {T.T }}$ & CFD \\
\hline $\begin{array}{l}\text { Original Design Space } \\
\text { (Cycle 1) }\end{array}$ & 203 -data & 0.791 & 0.200 & 2.000 & 0.441 & 2.000 & 1.250 & 2.000 & 1.042 & N/A \\
\hline $\begin{array}{l}\text { Extended Design Space } \\
\text { (Cycle 2) }\end{array}$ & 219 -data & 0.791 & 0.200 & 2.000 & 0.441 & 2.000 & 1.350 & 2.500 & 1.044 & 1.031 \\
\hline $\begin{array}{l}\text { NN-Enhanced Design Space } \\
\text { Cycle 3) }\end{array}$ & 306 -data & 0.791 & 0.200 & 2.000 & 0.441 & 2.000 & 1.350 & 2.500 & 1.043 & 1.031 \\
\hline
\end{tabular}


Table 8. Detailed Design Variables for the first blade (All variables are normalized by baseline values)

\begin{tabular}{|l|c|c|}
\hline Design Variable & Lower Limit & Upper Limit \\
\hline H/L & -100.0 & 200.0 \\
\hline Betal & 0.9 & 1.1 \\
\hline Uncovered Turning & 0.4 & 1.3 \\
\hline L1 & 0.1 & 1.1 \\
\hline L2F & 0.5 & 1.8 \\
\hline L3R & 0.5 & 2.6 \\
\hline L4 & 0.3 & 1.7 \\
\hline L5 & 1.0 & 2.1 \\
\hline L7 & 0.7 & 1.4 \\
\hline Channel Convergence Ratio & 1.0 & 1.1 \\
\hline Circle Factor & 0.1 & 5.0 \\
\hline
\end{tabular}

Table 9. Quality the Polynomial Response Surface Model for the first blade

\begin{tabular}{|l|c|c|c|c|}
\hline Summary of Fit & RSquare & $\begin{array}{c}\text { RSquare } \\
\text { Adj }\end{array}$ & $\begin{array}{c}\text { Root Mean } \\
\text { Square Error }\end{array}$ & $\begin{array}{c}\text { Observations (or } \\
\text { Sum Wgts) }\end{array}$ \\
\hline $\begin{array}{l}\text { Quadratic Model without NN- } \\
\text { data (Plain CFD data) (Cycle 1) }\end{array}$ & 0.78 & 0.57 & $3.82 \%$ & 160 \\
\hline $\begin{array}{l}\text { Quadratic Model (NN- } \\
\text { Enhanced data) (Cycle 2) }\end{array}$ & 0.87 & 0.86 & $1.74 \%$ & 2343 \\
\hline $\begin{array}{l}\text { Reduced Cubic Model (NN- } \\
\text { Enhanced data) (Cycle 3) }\end{array}$ & 0.97 & 0.97 & $0.81 \%$ & 2343 \\
\hline
\end{tabular}

Table 10. Summary of the revised design variables and ranges for the first blade (All design parameters are normalized by the baseline values).

\begin{tabular}{|l|c|c|}
\hline Design Variable & Lower Limit & Upper Limit \\
\hline H/L & -25.00 & -25.00 \\
\hline Beta1 & 0.86 & 0.99 \\
\hline Uncovered Turning & 0.63 & 0.63 \\
\hline L1 & 0.25 & 0.67 \\
\hline L2F & 0.91 & 1.82 \\
\hline L3R & 1.55 & 1.55 \\
\hline L4 & 0.68 & 0.68 \\
\hline L5 & 1103 & 1.92 \\
\hline L7 & 103 & 0.43 \\
\hline Channel Convergence Ratio & 1100 & 1.13 \\
\hline Circle Factor & 3.78 & 3.78 \\
\hline
\end{tabular}

Table 11. Optimum cases for the first blade obtained by reduced cubic model with NN-enhanced data for modified variable ranges (All design parameters and $\eta_{T-T}$ are normalized by the baseline values).

\begin{tabular}{|c|c|c|c|c|c|c|c|c|c|c|c|c|c|}
\hline & H/L & Betal & $\begin{array}{c}\text { Unc. } \\
\text { Turn }\end{array}$ & L1 & L2 & L3 & L4 & L5 & L7 & $\begin{array}{c}\text { Chan } \\
\text { CR }\end{array}$ & $\begin{array}{c}\text { Circ. } \\
\text { Fac }\end{array}$ & $\begin{array}{c}\eta_{\text {T-T }} \\
\text { RSM }\end{array}$ & $\begin{array}{c}\eta_{\text {T-r }} \\
\text { CFD }\end{array}$ \\
\hline Blade-1 & -25.00 & 0.94 & 0.63 & 0.59 & 1.48 & 1.55 & 0.68 & 1.78 & 0.66 & 1.04 & 3.78 & 1.04 & 1.05 \\
\hline Blade-2 & -25.00 & 0.99 & 0.63 & 0.67 & 1.36 & 1.60 & 0.67 & 1.44 & 0.76 & 1.06 & 3.80 & 1.07 & 1.04 \\
\hline
\end{tabular}




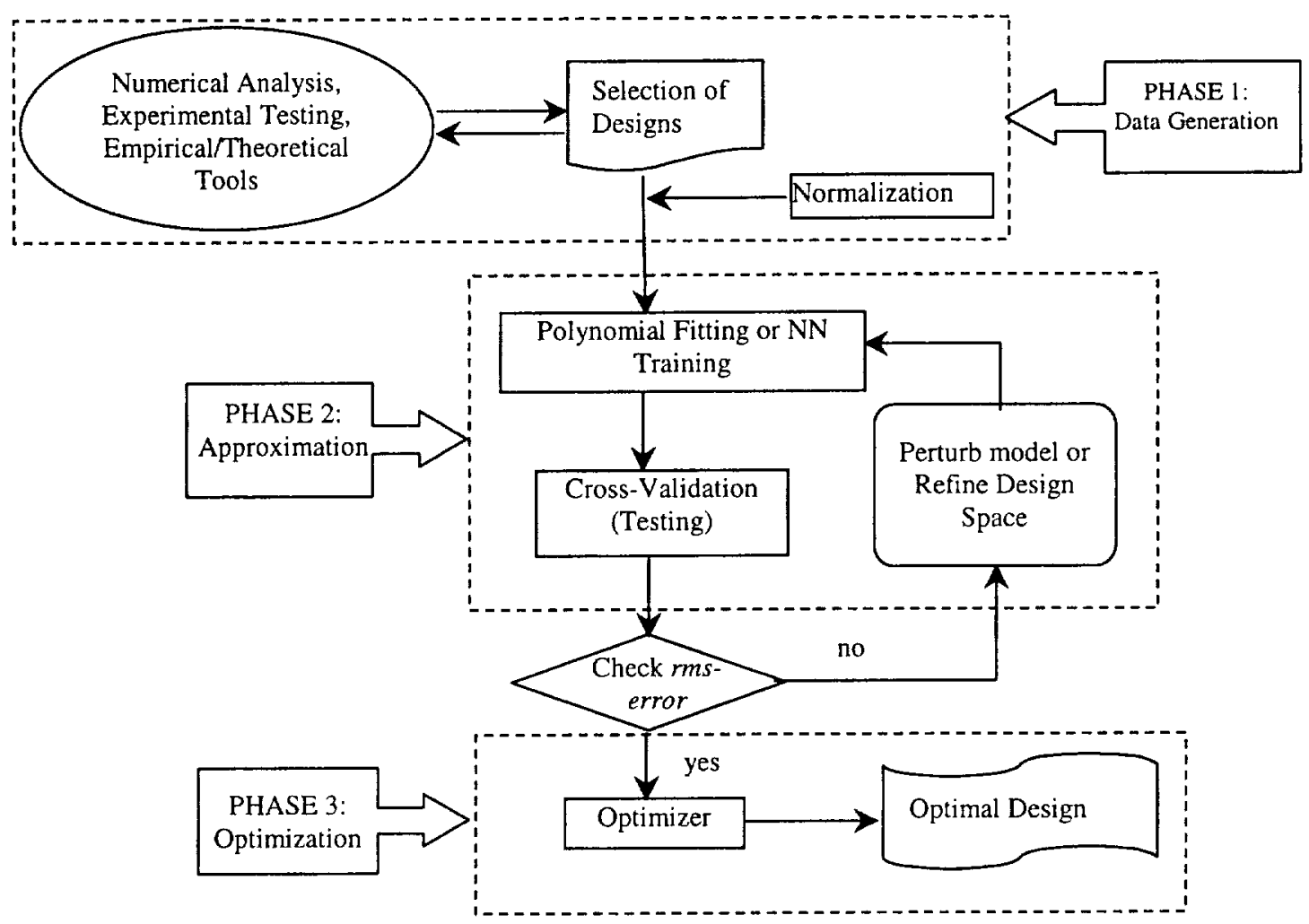

Figure 1. Schematic of the Global Optimization Procedure

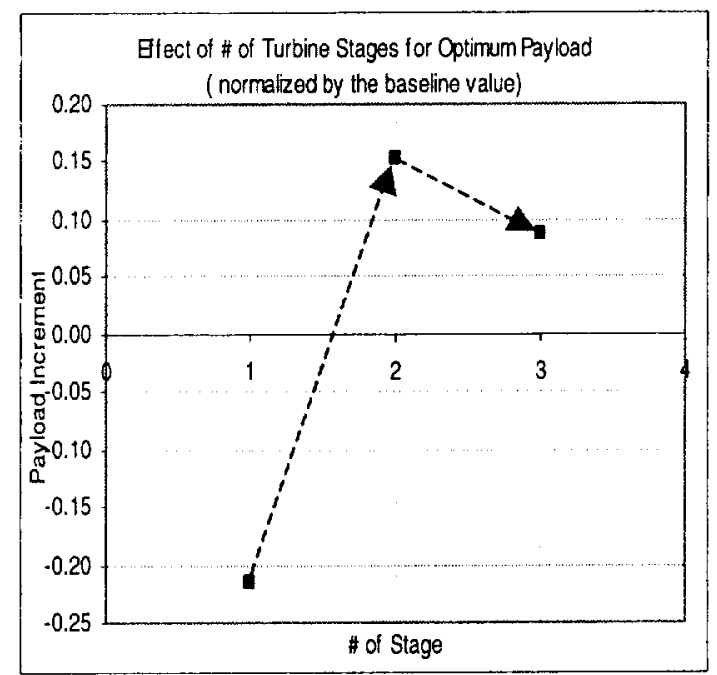

Figure 2. Effect of the number of turbine stage on optimum payload increment normalized by the baseline values 


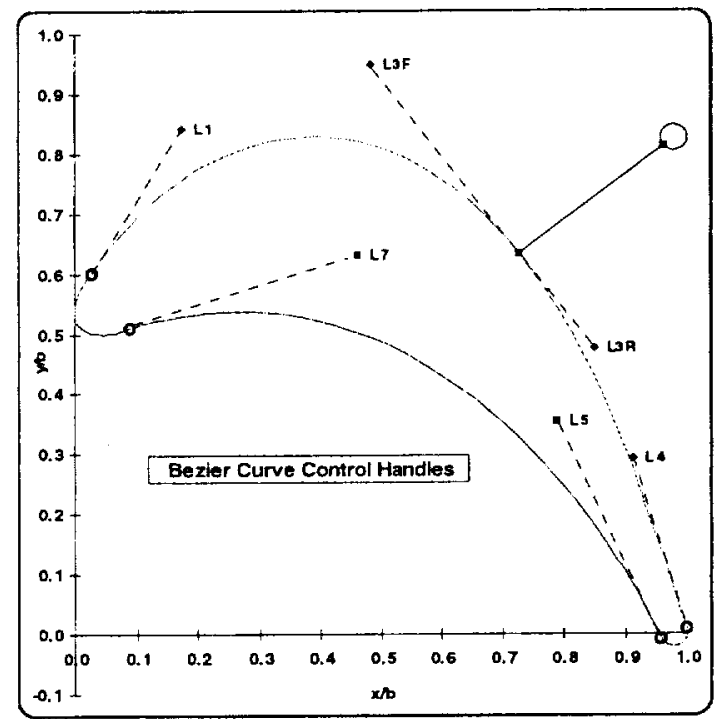

Figure 3. Bezier control handles for the airfoil.

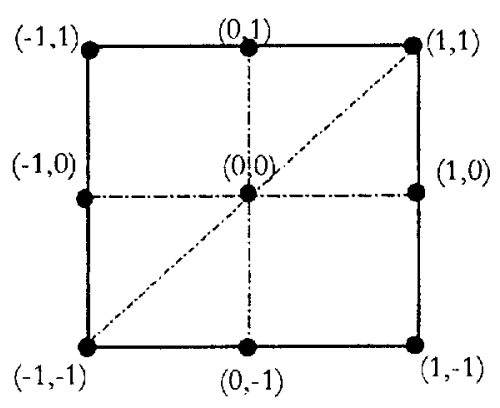

(a) $\mathrm{FCCD}$ in $2 \mathrm{D}$

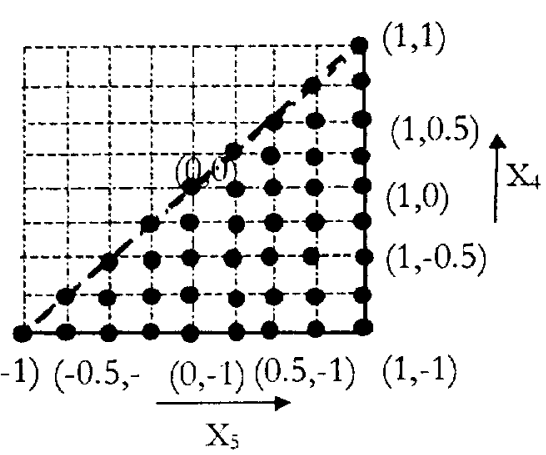

(b) Enhanced FCCD in 2D

Figure 4. Design of Experiment for the first vane

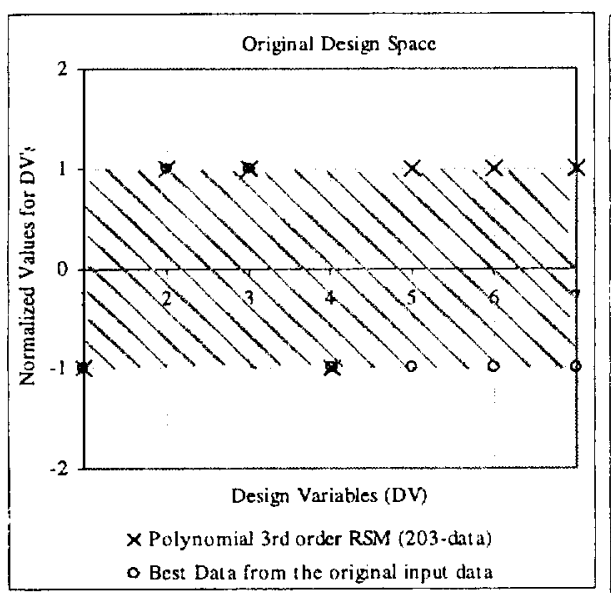

(a)

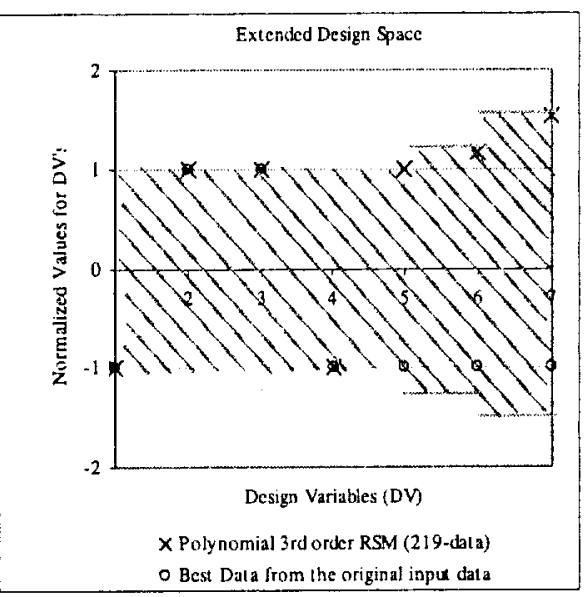

(b)

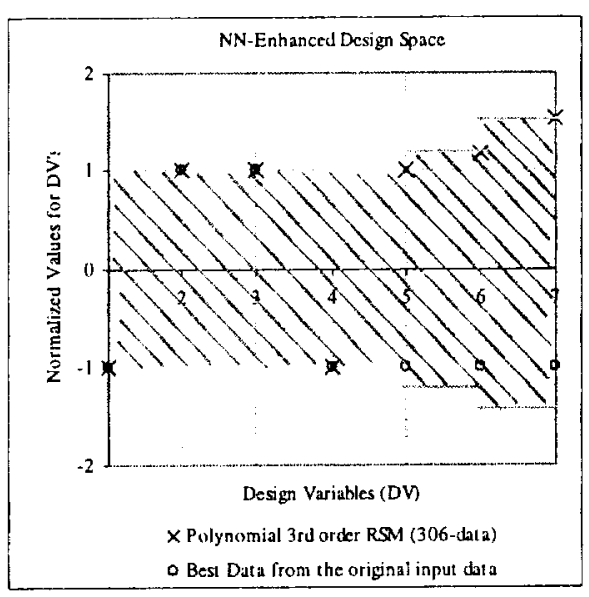

(c)

Figure 5. Comparison of the optimum design variables (DV) for the first vane (a) Original Design Space (Cycle 1) (where the design variables are bounded between -1 and +1 ), (b) Extended Design Space (Cycle 2), and (c) NNEnhanced Design Space (Cycle 3). 


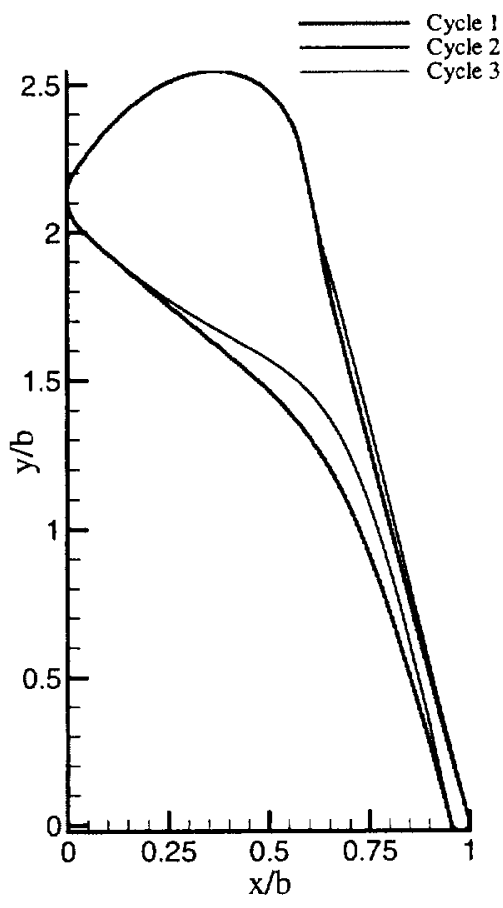

(a)

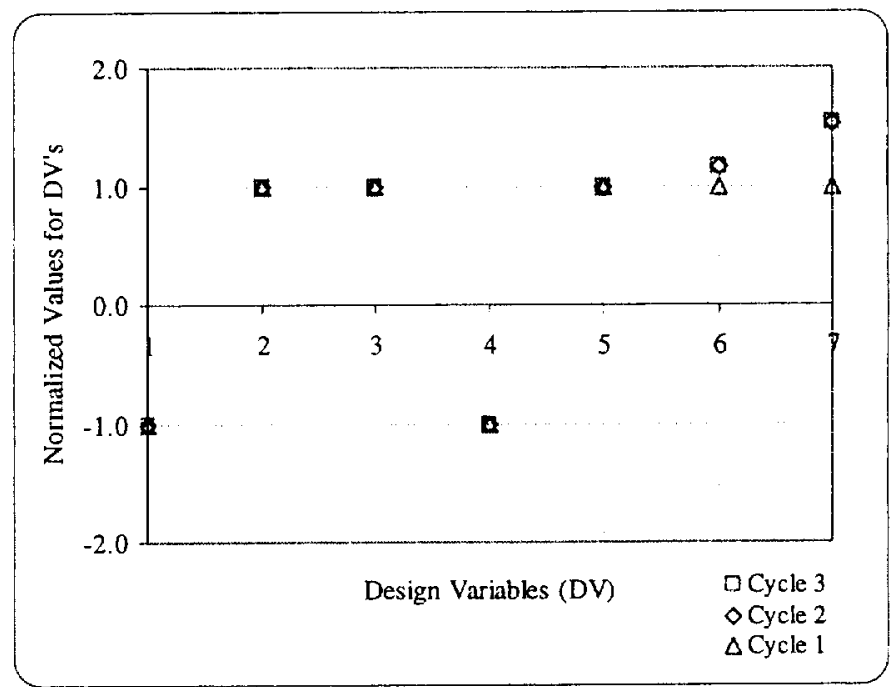

(b)

Figure 6. Summary of the three optimization cycles for the first vane (a) Optimal cross sections (Optimal designs of Cycles 2 and 3 coincide since they converge to the same configuration), and (b) Optimum design variables (where -1 and +1 show the minimum and maximum values of the design variables in the original design space, respectively).

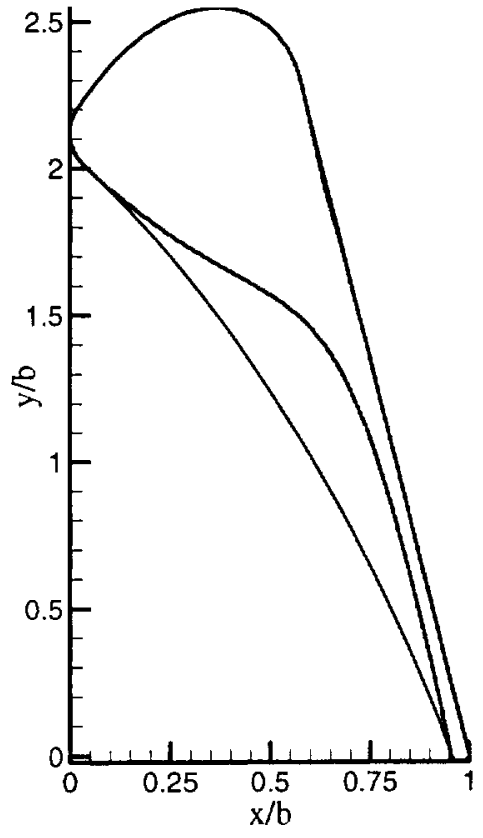

Figure 7. Illustration of multiple possibilities for the first vane. Both designs offer comparable efficiency, but the thicker profile is preferred from the structural considerations.
Efficiency contours for 203-data around highest efficiency

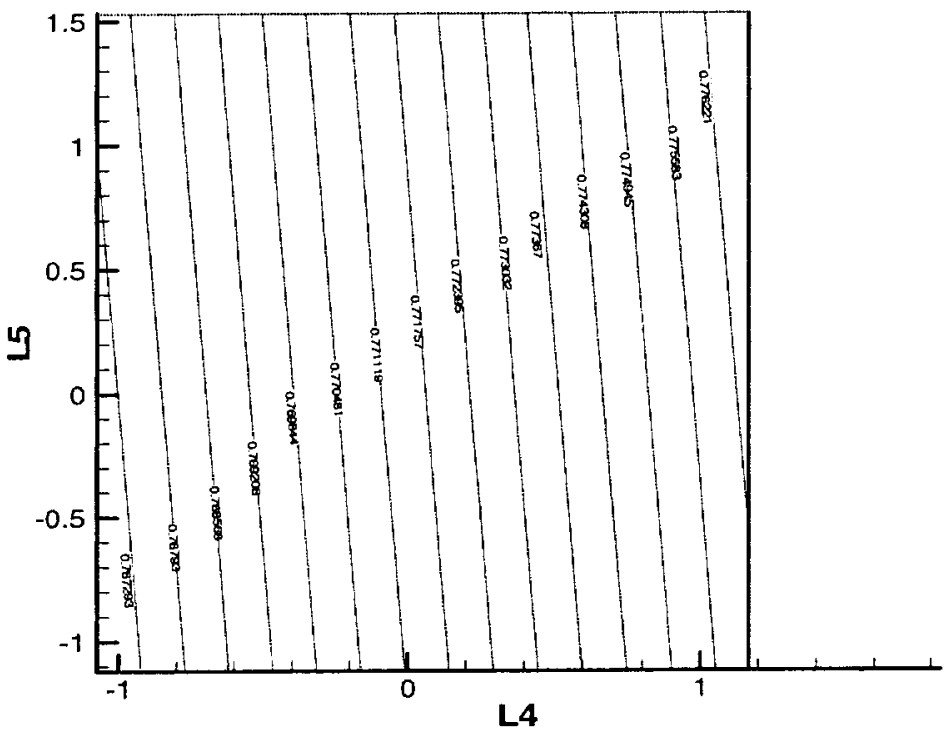

Figure 8. Efficiency contours for 203 data of the Cycle 1, which shows that $L 5$ has very little impact on the performance of the first vane. 


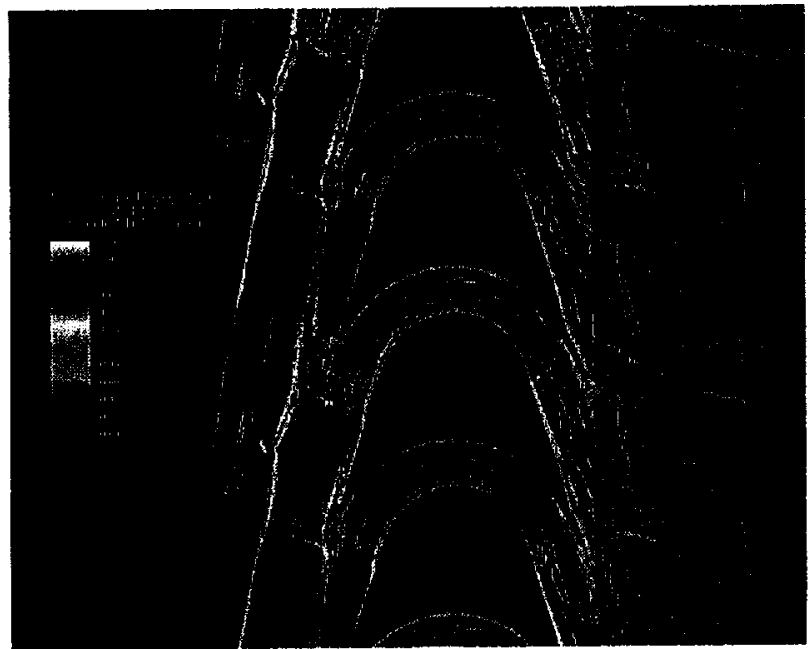

(a)

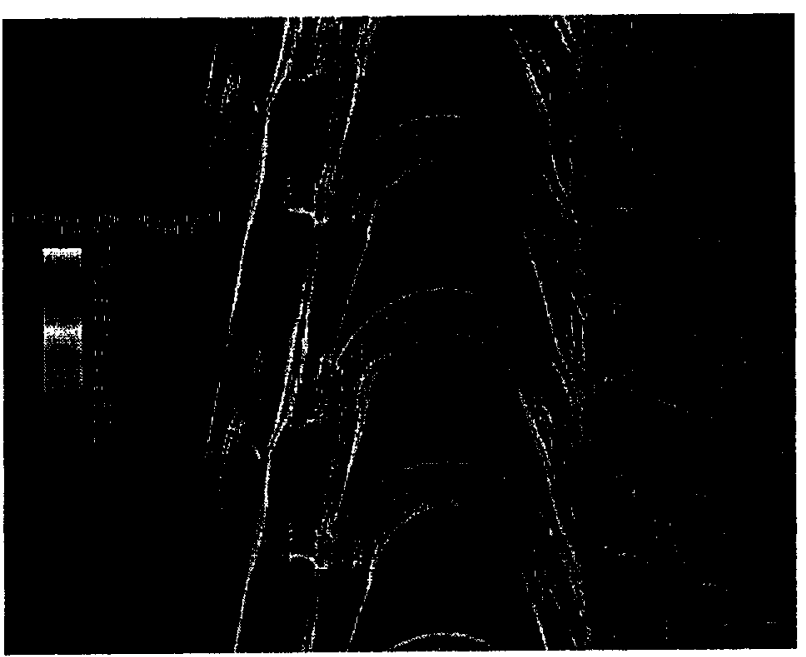

(b)

Figure 9. Instantaneous absolute Mach number contours for two first vane designs of essentially equal performances.

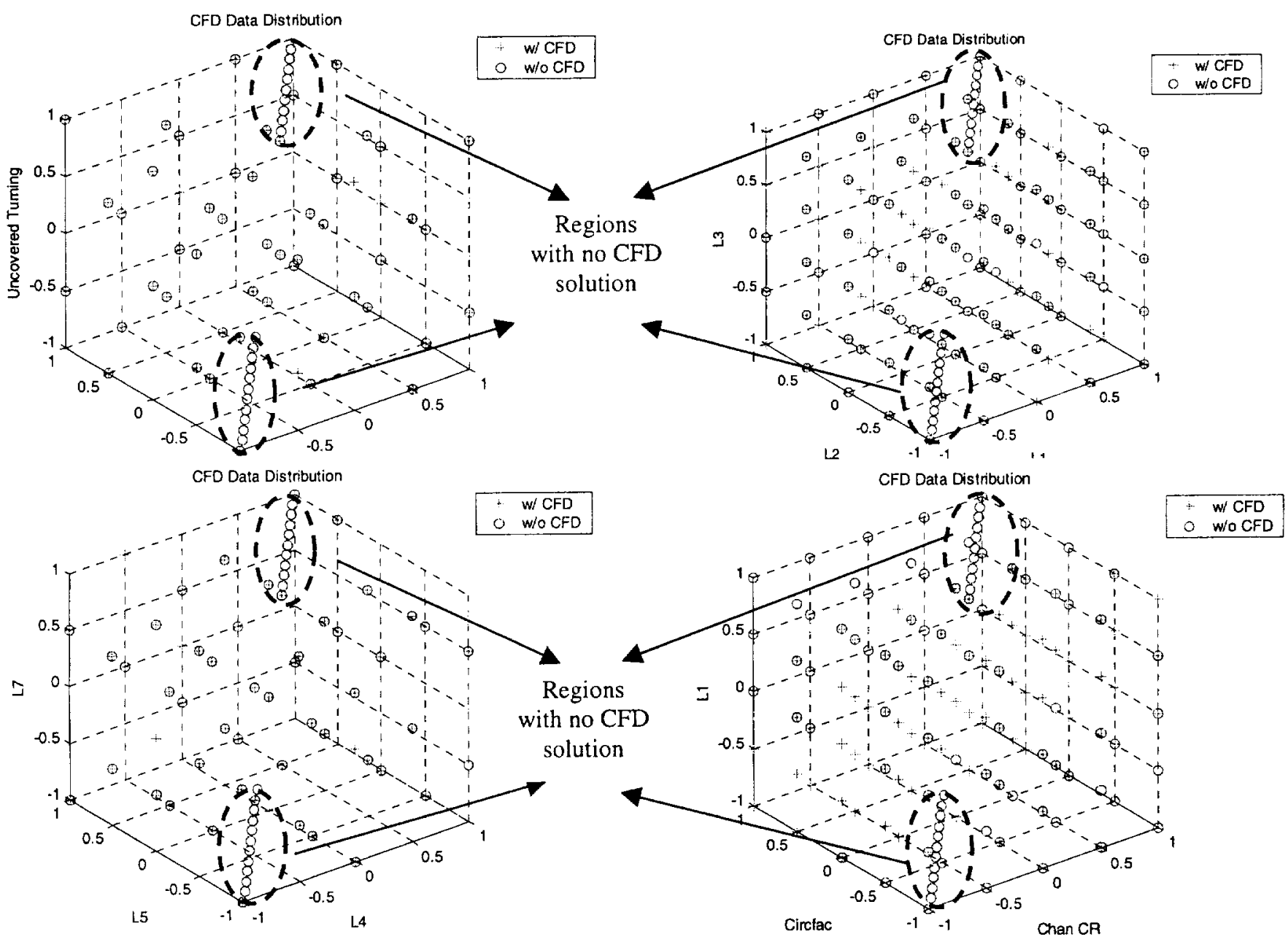

Figure 10. Summary of the cases for both successful and unsuccessful CFD simulations for the first blade. 


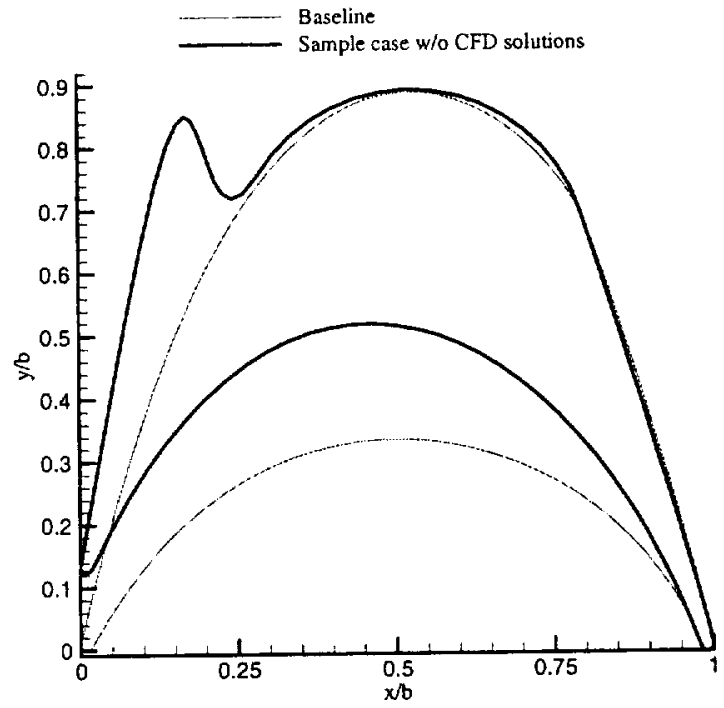

Figure 11. Sample Case without CFD solutions from Design Space of the first blade.

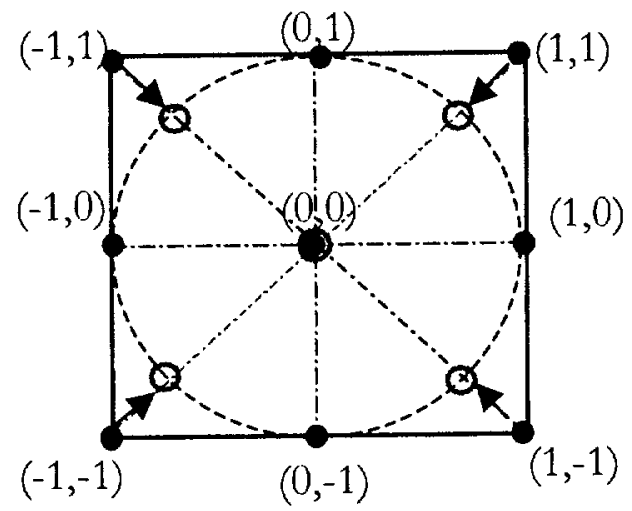

Figure 12. Schematic of the additional data generated for the first blade in $2 \mathrm{D}$.

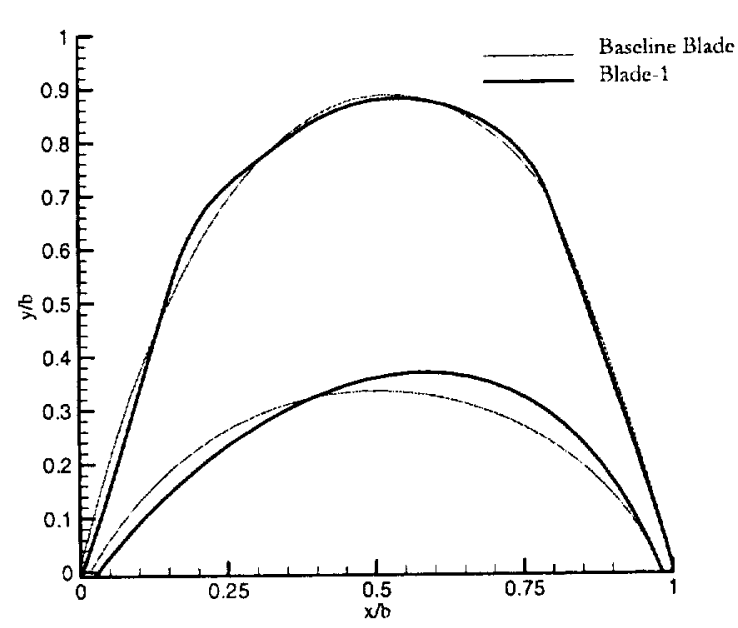

(a) Blade-1

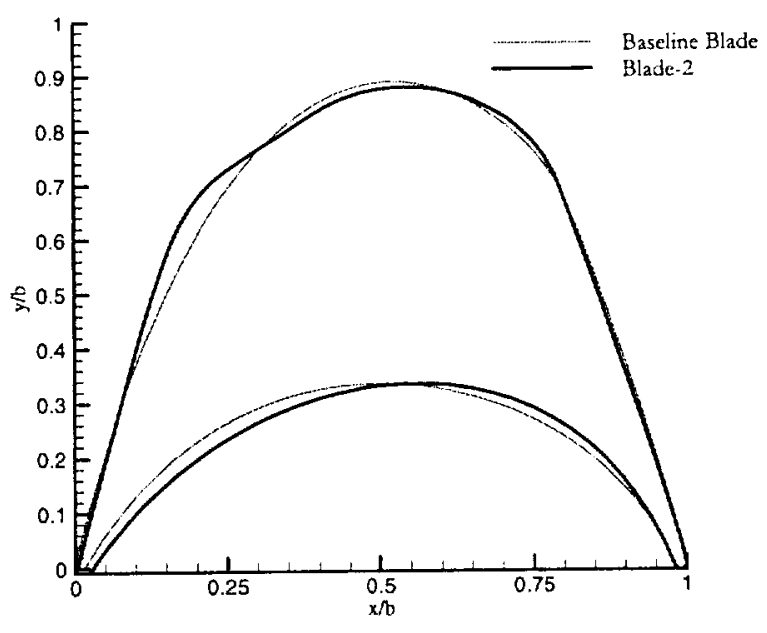

(b) Blade-2

Figure 13. Optimum Shapes for the first blade obtained by reduced cubic model with NN-enhanced data for modified variable ranges defined in Table 1. 